\title{
Further Discussion on the Calculation of Fourier Series
}

\author{
Caixia Zhang \\ College of Mathematics and Statistics, Northeast Petroleum University, Daqing, China \\ Email: zhangcai6476@163.com
}

Received 9 February 2015; accepted 23 March 2015; published 24 March 2015

Copyright (C) 2015 by author and Scientific Research Publishing Inc.

This work is licensed under the Creative Commons Attribution International License (CC BY). http://creativecommons.org/licenses/by/4.0/

(c) (i) Open Access

\section{Abstract}

Fourier series is an important mathematical concept. It is well known that we need too much computation to expand the function into Fourier series. The existing literature only pointed that its Fourier series is sine series when the function is an odd function and its Fourier series is cosine series when the function is an even function. And on this basis, in this paper, according to the function which satisfies different conditions, we give the different forms of Fourier series and the specific calculation formula of Fourier coefficients, so as to avoid unnecessary calculation. In addition, if a function is defined on $[0, a]$, we can make it have some kind of nature by using the extension method as needed. So we can get the corresponding form of Fourier series.

\section{Keywords}

Fourier Coefficients, Fouries Series, Period, Series Expansion, Extension

\section{Preliminary Knowledge}

Definition 1 [1]-[3] Let $f(x)$ be an integrable function on $[-l, l]$. Then the coefficients $a_{n}$ and $b_{n}$ are calculated by

$$
\begin{aligned}
& a_{n}=\frac{1}{l} \int_{-l}^{l} f(x) \cos \frac{n \pi x}{l} d x, \quad(n=0,1,2, \cdots) ; \\
& b_{n}=\frac{1}{l} \int_{-l}^{l} f(x) \sin \frac{n \pi x}{l} d x, \quad(n=1,2, \cdots) .
\end{aligned}
$$

and are called the Fourier coefficients of $f(x)$.

Definition $2[1]$-[5] Let $f(x)$ with the period $2 l$ be an integrable function on $[-l, l]$, trigonometric series 
with the Fourier coefficient are called Fourier series of $f(x)$, denoted by

$$
f(x) \sim \frac{a_{0}}{2}+\sum_{n=1}^{\infty}\left(a_{n} \cos \frac{n \pi x}{l}+b_{n} \sin \frac{n \pi x}{l}\right)
$$

Lemma 1 [6] Let $f(x)$ be an integrable function on $[-l, l]$ with period of $2 l$, the Fourier coefficients are calculated according to period of $2 k l\left(k \in N^{+}\right)$. The calculation indicates there are same results between Fourier series with period of $2 l$ and $2 k l\left(k \in N^{+}\right)$.

\section{Calculating Fourier Series According to the Nature of the Function}

Theorem 1 Let $f(x)$ be an integrable function on $[-l, l]$ and satisfy the condition $f(x+l)=-f(x)$, then we have

$$
f(x) \sim \sum_{k=1}^{\infty}\left[a_{2 k-1} \cos \frac{(2 k-1) \pi x}{l}+b_{2 k-1} \sin \frac{(2 k-1) \pi x}{l}\right]
$$

where $a_{2 k-1}=\frac{2}{l} \int_{0}^{l} f(x) \cos \frac{(2 k-1) \pi x}{l} \mathrm{~d} x, \quad b_{2 k-1}=\frac{2}{l} \int_{0}^{l} f(x) \sin \frac{(2 k-1) \pi x}{l} \mathrm{~d} x, \quad k=1,2, \cdots$.

Proof It was clear that the period of $f(x)$ is $2 l$ and we have

$$
a_{0}=\frac{1}{l} \int_{-l}^{l} f(x) \mathrm{d} x=\frac{1}{l}\left[\int_{-l}^{0} f(x) \mathrm{d} x+\int_{0}^{l} f(x) \mathrm{d} x\right]
$$

Let $x=t-l$, then $\mathrm{d} x=\mathrm{d} t$. Therefore

$$
\int_{-l}^{0} f(x) d x=\int_{0}^{l} f(t-l) \mathrm{d} t=\int_{0}^{l}\{-f[(t-l)+l]\} \mathrm{d} t=-\int_{0}^{l} f(t) \mathrm{d} t=-\int_{0}^{l} f(x) \mathrm{d} x .
$$

So we get

$a_{0}=0$.

$$
a_{n}=\frac{1}{l} \int_{-l}^{l} f(x) \cos \frac{n \pi x}{l} \mathrm{~d} x=\frac{1}{l}\left[\int_{-l}^{0} f(x) \cos \frac{n \pi x}{l} \mathrm{~d} x+\int_{0}^{l} f(x) \cos \frac{n \pi x}{l} \mathrm{~d} x\right] .
$$

Let $x=t-l$, then $\mathrm{d} x=\mathrm{d} t$. Therefore

$$
\begin{aligned}
\int_{-l}^{0} f(x) \cos \frac{n \pi x}{l} \mathrm{~d} x & =\int_{0}^{l} f(t-l) \cos \frac{n \pi(t-l)}{l} \mathrm{~d} t=\int_{0}^{l}\{-f[(t-l)+l]\} \cos \left(\frac{n \pi t}{l}-n \pi\right) \mathrm{d} t \\
& =-\int_{0}^{l} f(t)(-1)^{n} \cos \frac{n \pi t}{l} \mathrm{~d} t=(-1)^{n+1} \int_{0}^{l} f(x) \cos \frac{n \pi x}{l} \mathrm{~d} x .
\end{aligned}
$$

Therefore, we obtain

$$
\begin{aligned}
a_{n} & =\frac{1}{l}\left[(-1)^{n+1}+1\right] \int_{0}^{l} f(x) \cos \frac{n \pi x}{l} \mathrm{~d} x \\
& =\left\{\begin{array}{rrr}
0, & n=2 k ; \\
\frac{2}{l} \int_{0}^{l} f(x) \cos \frac{(2 k-1) \pi x}{l} \mathrm{~d} x, & n=2 k-1 .
\end{array} \quad k=1,2, \cdots .\right.
\end{aligned}
$$

In the same way, we have

$$
b_{n}=\left\{\begin{array}{ll}
0, & n=2 k ; \\
\frac{2}{l} \int_{0}^{l} f(x) \sin \frac{(2 k-1) \pi x}{l} \mathrm{~d} x, & n=2 k-1 .
\end{array} \quad k=1,2, \cdots .\right.
$$

In a word, while $n$ is an even number, $a_{n}=b_{n}=0$, and 


$$
\begin{aligned}
& a_{2 k-1}=\frac{2}{l} \int_{0}^{l} f(x) \cos \frac{(2 k-1) \pi x}{l} \mathrm{~d} x, \quad k=1,2, \cdots . \\
& b_{2 k-1}=\frac{2}{l} \int_{0}^{l} f(x) \sin \frac{(2 k-1) \pi x}{l} \mathrm{~d} x,
\end{aligned}
$$

Thus in this case, the expansion reduces to

$$
f(x) \sim \sum_{k=1}^{\infty}\left[a_{2 k-1} \cos \frac{(2 k-1) \pi x}{l}+b_{2 k-1} \sin \frac{(2 k-1) \pi x}{l}\right]
$$

Theorem 2 Let $f(x)$ be an integrable function on $[-l, l]$ and satisfy the conditions $f(x+l)=f(x)$, then we have

$$
f(x) \sim \frac{a_{0}}{2}+\sum_{k=1}^{\infty}\left[a_{2 k} \cos \frac{2 k \pi x}{l}+b_{2 k} \sin \frac{2 k \pi x}{l}\right]
$$

where $a_{2 k}=\frac{2}{l} \int_{0}^{l} f(x) \cos \frac{2 k \pi x}{l} \mathrm{~d} x, \quad k=0,1,2, \cdots, \quad b_{2 k}=\frac{2}{l} \int_{0}^{l} f(x) \sin \frac{2 k \pi x}{l} \mathrm{~d} x, \quad k=1,2, \cdots$.

Proof The period of $f(x)$ is $l$, so we can calculate Fouries series of $f(x)$ with period of $2 l$ by Lemma 1 . We have

$$
a_{0}=\frac{1}{l} \int_{-l}^{l} f(x) \mathrm{d} x=\frac{1}{l}\left[\int_{-l}^{0} f(x) \mathrm{d} x+\int_{0}^{l} f(x) \mathrm{d} x\right]
$$

Let $x=t-l$, then $\mathrm{d} x=\mathrm{d} t$. Therefore

$$
\int_{-l}^{0} f(x) \mathrm{d} x=\int_{0}^{l} f(t-l) \mathrm{d} t=\int_{0}^{l} f(t) \mathrm{d} t
$$

So we get

$$
\begin{aligned}
& a_{0}=\frac{2}{l} \int_{0}^{l} f(x) \mathrm{d} x, \\
& a_{n}=\frac{1}{l} \int_{-l}^{l} f(x) \cos \frac{n \pi x}{l} \mathrm{~d} x=\frac{1}{l}\left[\int_{-l}^{0} f(x) \cos \frac{n \pi x}{l} \mathrm{~d} x+\int_{0}^{l} f(x) \cos \frac{n \pi x}{l} \mathrm{~d} x\right] .
\end{aligned}
$$

Let $x=t-l$, then $\mathrm{d} x=\mathrm{d} t$. Therefore

$$
\begin{aligned}
\int_{-l}^{0} f(x) \cos \frac{n \pi x}{l} \mathrm{~d} x & =\int_{0}^{l} f(t-l) \cos \frac{n \pi(t-l)}{l} \mathrm{~d} t=\int_{0}^{l} f(t) \cos \left(\frac{n \pi t}{l}-n \pi\right) \mathrm{d} t \\
& =\int_{0}^{l} f(t)(-1)^{n} \cos \frac{n \pi t}{l} \mathrm{~d} t=(-1)^{n} \int_{0}^{l} f(x) \cos \frac{n \pi x}{l} \mathrm{~d} x,
\end{aligned}
$$

We obtain

$$
\begin{aligned}
a_{n} & =\frac{1}{l}\left[(-1)^{n}+1\right] \int_{0}^{l} f(x) \cos \frac{n \pi x}{l} \mathrm{~d} x \\
& = \begin{cases}0, & n=2 k-1 ; \\
\frac{2}{l} \int_{0}^{l} f(x) \cos \frac{2 k \pi x}{l} \mathrm{~d} x, & n=2 k .\end{cases}
\end{aligned}
$$

In the same way, we have

$$
b_{n}=\left\{\begin{array}{ll}
0, & n=2 k-1 ; \\
\frac{2}{l} \int_{0}^{l} f(x) \sin \frac{2 k \pi x}{l} \mathrm{~d} x, & n=2 k .
\end{array} \quad k=1,2, \cdots .\right.
$$

Thus in this case, the expansion reduces to 


$$
f(x) \sim \frac{a_{0}}{2}+\sum_{k=1}^{\infty}\left[a_{2 k} \cos \frac{2 k \pi x}{l}+b_{2 k} \sin \frac{2 k \pi x}{l}\right]
$$

where $\quad a_{2 k}=\frac{2}{l} \int_{0}^{l} f(x) \cos \frac{2 k \pi x}{l} \mathrm{~d} x, \quad k=0,1,2, \cdots, \quad b_{2 k}=\frac{2}{l} \int_{0}^{l} f(x) \sin \frac{2 k \pi x}{l} \mathrm{~d} x, \quad k=1,2, \cdots$.

Theorem 3 Let $f(x)$ be an integrable function on $[0, l]$. When $\frac{l}{2}<x \leq l$, it satisfies the condition $f(x)=-f(l-x)$. Then we have

(1) While $f(x)$ is an even function in $(-l, l)$, then we get

$$
f(x) \sim \sum_{k=1}^{\infty} a_{2 k-1} \cos \frac{(2 k-1) \pi x}{l}
$$

where $a_{2 k-1}=\frac{4}{l} \int_{0}^{\frac{l}{2}} f(x) \cos \frac{(2 k-1) \pi x}{l} \mathrm{~d} x, \quad k=1,2, \cdots$.

(2) While $f(x)$ is an odd function in $(-l, l)$, then we get

$$
f(x) \sim \sum_{k=1}^{\infty} b_{2 k} \sin \frac{2 k \pi x}{l}
$$

where $b_{2 k}=\frac{4}{l} \int_{0}^{\frac{l}{2}} f(x) \sin \frac{2 k \pi x}{l} \mathrm{~d} x, \quad k=1,2, \cdots$.

Proof (1) We use the method of periodic extension to $f(x)$ with period of $2 l$.

Because $f(x)$ is an even function, we have $b_{n}=0, n=1,2, \cdots$.

$$
a_{0}=\frac{2}{l} \int_{0}^{l} f(x) \mathrm{d} x=\frac{2}{l}\left[\int_{0}^{\frac{l}{2}} f(x) \mathrm{d} x+\int_{\frac{1}{2}}^{l} f(x) \mathrm{d} x\right]=\frac{2}{l}\left[\int_{0}^{\frac{l}{2}} f(x) \mathrm{d} x-\int_{\frac{1}{2}}^{l} f(l-x) \mathrm{d} x\right]
$$

Let $t=l-x, \int_{\frac{1}{2}}^{l} f(l-x) \mathrm{d} x=\int_{0}^{\frac{l}{2}} f(t) \mathrm{d} t$, then we have

$$
\begin{aligned}
& a_{0}=0 \\
& a_{n}=\frac{2}{l} \int_{0}^{l} f(x) \cos \frac{n \pi x}{l} \mathrm{~d} x=\frac{2}{l}\left[\int_{0}^{\frac{l}{2}} f(x) \cos \frac{n \pi x}{l} \mathrm{~d} x-\int_{\frac{1}{2}}^{l} f(l-x) \cos \frac{n \pi x}{l} \mathrm{~d} x\right] .
\end{aligned}
$$

Let $t=l-x$, therefore

$$
\int_{\frac{l}{2}}^{l} f(l-x) \cos \frac{n \pi x}{l} \mathrm{~d} x=-\int_{\frac{1}{2}}^{0} f(t) \cos \frac{n \pi(l-t)}{l} \mathrm{~d} t=(-1)^{n} \int_{0}^{\frac{l}{2}} f(t) \cos \frac{n \pi t}{l} \mathrm{~d} t
$$

We obtain

$$
\begin{aligned}
a_{n} & =\left[1-(-1)^{n}\right] \frac{2}{l} \int_{0}^{\frac{l}{2}} f(x) \cos \frac{n \pi x}{l} \mathrm{~d} x \\
& = \begin{cases}0, & n=2 k ; \\
\frac{4}{l} \int_{0}^{\frac{l}{2}} f(x) \cos \frac{(2 k-1) \pi x}{l} \mathrm{~d} x, & n=2 k-1 .\end{cases}
\end{aligned}
$$

Thus in this case, the expansion reduces to

$$
f(x) \sim \sum_{k=1}^{\infty} a_{2 k-1} \cos \frac{(2 k-1) \pi x}{l}
$$

where $a_{2 k-1}=\frac{4}{l} \int_{0}^{\frac{1}{2}} f(x) \cos \frac{(2 k-1) \pi x}{l} \mathrm{~d} x, \quad k=1,2, \cdots$. 
(2) In the same way, we can prove Theorem 3 (2).

Similarly, we can prove the following Theorem 4.

Theorem 4 Let $f(x)$ be an integrable function on $[0, l]$. When $\frac{l}{2}<x \leq l$, it satisfies the condition $f(x)=f(l-x)$. Then we have

(1) While $f(x)$ is an even function in $(-l, l)$, then we get

$$
f(x) \sim \sum_{k=1}^{\infty} a_{2 k} \cos \frac{2 k \pi x}{l}
$$

where $a_{2 k}=\frac{4}{l} \int_{0}^{\frac{l}{2}} f(x) \cos \frac{2 k \pi x}{l} \mathrm{~d} x, \quad k=0,1,2, \cdots$.

(2) While $f(x)$ is an odd function in $(-l, l)$, then we get

$$
f(x) \sim \sum_{k=1}^{\infty} b_{2 k-1} \sin \frac{(2 k-1) \pi x}{l}
$$

where $b_{2 k-1}=\frac{4}{l} \int_{0}^{\frac{l}{2}} f(x) \sin \frac{(2 k-1) \pi x}{l} \mathrm{~d} x, \quad k=1,2, \cdots$.

\section{Conclusion}

Suppose the function is defined on $\left[0, \frac{l}{2}\right]$, if we use symmetry extension about the point $\left(\frac{l}{2}, 0\right)$ and then use odd and periodic extension, we can get two forms of Fourier series as Theorem 3. If we use symmetry extension about the line $x=\frac{l}{2}$ and then use odd and periodic extension, we can get two forms of Fourier series as Theorem 4. Suppose the function is defined on $\left[-\frac{l}{2}, 0\right]$, we have a similar conclusion.

\section{Acknowledgements}

I would like to thank the referees and the editor for their valuable suggestions.

\section{References}

[1] Department of Mathematics of East China Normal University (2010) Mathematical Analysis. 4th Edition, Higher Education Press, Beijing, 62-72. (In Chinese)

[2] He, G.Z. (2008) Discussion on a Style of Fourier Series Expansion. Journal of Leshan Teachers College, 23, 27-28. (In Chinese)

[3] Zheng, C. and Qiu, W.G. (2010) Integration Techniques Based on Fourier. Studies in College Mathematics, 13, 31-32. (In Chinese)

[4] Wang, B.Y. and Qi, X.S. (2011) Two Methods for Summing Trigonometric Series. Studies in College Mathematics, 14, 33-34. (In Chinese)

[5] Jiao, H.Y. and Liu, W.H. (2011) Fourier Expansion and a Class of Series. Studies in College Mathematics, 14, 35-36. (In Chinese)

[6] Ding, X.H. (2004) Several Problems of Fouries Series Expansion. Journal of Daxian Teachers College (Natural Science Edition), 14, 1-4. (In Chinese) 